\title{
Correction to: Modeling hallmark pathology using motor neurons derived from the family and sporadic amyotrophic lateral sclerosis patient-specific iPS cells
}

Xuejiao Sun ${ }^{1}$, Jianyuan Song ${ }^{2}$, Hailong Huang ${ }^{1}$, Hong Chen ${ }^{1}$ and Kun Qian ${ }^{2 *}$

\section{Correction to: Stem Cell Res Ther}

https://doi.org/10.1186/s13287-018-1048-1

The original article [1] was submitted and published without co-author Hong Chen's permission. The author, Hong Chen has therefore requested to have her name disregarded from the author list. All of the authors agree with this change.

\section{Author details \\ ${ }^{1}$ Department of Rehabilitation Medicine, Tongji Hospital, Tongji Medical College, Huazhong University of Science and Technology, Jiefang Avenue 1095, Wuhan 430030, China. ${ }^{2}$ Reproductive Medicine Center, Tongji Hospital, Tongji Medicine College, Huazhong University of Science and Technology, Jiefang Avenue 1095, Wuhan 430030, China.}

Received: 6 March 2019 Revised: 6 March 2019

Accepted: 7 March 2019 Published online: 15 March 2019

\section{Reference}

1. Sun X, Song J, Huang H, Chen H, Qian K. Modeling hallmark pathology using motor neurons derived from the family and sporadic amyotrophic lateral sclerosis patient-specific iPS cells. Stem Cell Res Ther. 2018;9:315 https://doi.org/10.1186/s13287-018-1048-1.

\footnotetext{
* Correspondence: kunqian@tjh.tjmu.edu.cn

${ }^{2}$ Reproductive Medicine Center, Tongji Hospital, Tongji Medicine College, Huazhong University of Science and Technology, Jiefang Avenue 1095, Wuhan 430030, China

Full list of author information is available at the end of the article
} 